\title{
The Motivation-Based Promotion of Proactive Control: The Role of Salience Network
}

\begin{abstract}
Lei Qiao ${ }^{1,2}$, Lei X ${ }^{3}$, Xianwei Che ${ }^{4}$, Lijie Zhang ${ }^{2}$, Yadan $L^{5}{ }^{5}$, Gui Xue ${ }^{2,6}$, Hong $\mathrm{Li}^{1}$ and Antao Chen ${ }^{2 *}$

${ }^{1}$ College of Psychology and Sociology, Shenzhen University, Shenzhen, China, ${ }^{2}$ Key Laboratory of Cognition and Personality of Ministry of Education, Faculty of Psychology, Southwest University, Chongqing, China, ${ }^{3}$ Key Laboratory for Neuroinformation, Center for Information in Medicine, University of Electronic Science and Technology of China, Chengdu, China, ${ }^{4}$ Monash Alfred Psychiatry Research Centre, The Alfred and Central Clinical School, Monash University, Melbourne, VIC, Australia, ${ }^{5}$ MOE Key Laboratory of Modern Teaching Technology, Shaanxi Normal University, Xi'an, China, ${ }^{6}$ State Key Laboratory of Cognitive Neuroscience and Learning \& IDG/McGovern Institute for Brain Research, Beijing Normal University, Beijing, China
\end{abstract}

It has been shown that reward motivation can facilitate proactive control, a cognitive control mode that is characterized of prior preparation and sustained holding of the goal-relevant information in working memory. However, it remains to be established the neural networks that may be involved in this promotion effect. In this study, participants underwent the AX-Continuous Performance Task (AX-CPT) that measures relative proactive control during functional magnetic resonance imaging (fMRI) scanning. We employed independent component analysis to decompose multiple brain networks and identified the task related network. Results showed that the salience network (SN) was engaged in the AX-CPT protocol. Importantly, our data demonstrated that reward modulated the association between task engagement of $\mathrm{SN}$ and proactive control, whereby the positive correlation was particularly observed in the reward condition. Moreover, reward modulated task engagement of the $\mathrm{SN}$ in a proactive manner, which may contribute to the behavioral proactive performance. Overall, our data suggest the involvement of $\mathrm{SN}$ in the reward facilitation effect of proactive control.

Keywords: dual-mechanism of control, independent component analysis, AX-Continuous Performance Task, salience network, proactive control, reactive control, reward

\section{INTRODUCTION}

Cognitive control is defined as a set of processes that allow individuals to flexibly coordinate thoughts and behaviors in accordance with internal goals (Miller and Cohen, 2001). It has been widely studied how cognitive control influences our behavior and daily life. Among these studies, a theoretical framework-the dual-mechanism of control (DMC) theory-postulates that there are two distinct cognitive modes in cognitive processing, namely, reactive control and proactive control (Braver et al., 2007; Braver, 2012). In reactive control, task-relevant information (e.g., task instructions, goals, stimulus-response mapping) is processed in a transient manner (Braver, 2012). Reactive control is helpful to detect and resolve interference only when an interference occurs; thus, attention is recruited as a late correction mechanism. Meanwhile, in proactive control, task-relevant information is actively maintained in working memory to direct attention and possible responses. Proactive control is a sustained form of control, which can be engaged prior to the presentation of stimuli and can promote rapid and efficient responses (Jaspar et al., 2014; Chiew and Braver, 2016). 
Previous studies have suggested that reward motivation can facilitate goal-directed behavior via the adoption of an optimal cognitive strategy (Pochon et al., 2002; Gilbert and Fiez, 2004; Taylor et al., 2004; Small et al., 2005; Fröber and Dreisbach, 2014; Etzel et al., 2015). Indeed, the DMC theory proposes that reward motivation can reliably enhance a proactive control mode relative to reactive control. For instance, Locke and Braver (2008) compared reward and penalty effects on strategy transformation between proactive and reactive control. Results demonstrated that reward condition showed sustained activity of proactive control, whereby the penalty condition produced a shift toward a more reactive, probe-based pattern of activation. Jimura et al. (2010) also found that reward can promote proactive control, and that individuals with high reward sensitivity exhibited better working memory performance in rewarding contexts.

In the context of motivation and cognitive control, it is interesting to investigate how the brain networks may interact to support the motivational effects in control (Botvinick and Braver, 2015). Although the literature supports the idea that reward motivation can promote a proactive mode of cognitive control, little is known about the activity of neural networks that may underlie this process. A network perspective may provide insights of the synchronized brain activity and the dynamic information changes between brain regions that support the cognitive control modes (Papo et al., 2014). According to the literature, it is hypothesized that the fronto-parietal network (FPN) and/or the salience network (SN) would be involved in the incentive enhancement of proactive control as of their extensive involvement in reward and cognitive control.

The FPN, mainly composed of the lateral prefrontal cortex (lateral PFC) and the posterior parietal cortex (PPC), plays an important role in both cognitive control and reward. The FPN is engaged in various cognitive tasks in which cognitive control is needed to guide selective attention (Dosenbach et al., 2007, 2008; Cole et al., 2013). Meanwhile, reward motivation has been reported to enhance task coding in fronto-parietal cortex. Specifically, the authors trained a multi-voxel classifier in baseline session and used this classifier to distinguish different task sets in a reward session. They found that the classification accuracy was higher in reward trials than non-reward trials in fronto-parietal regions, and that the enhancement of the decoding accuracy mediated the improvement of behavioral performance (Etzel et al., 2015). Importantly, it has been suggested that the lateral PFC also plays an important role in the motivation-based enhancement of proactive control, in which proactive control was associated with sustained activity of the lateral PFC (Locke and Braver, 2008; Costumero et al., 2015).

Similarly, the SN is also involved in both cognitive control and reward. The SN is known to support the detection of behaviorally relevant stimuli (Uddin, 2014; Dajani and Uddin, 2015), the maintenance and implementation of task sets (Dosenbach et al., 2006; Nelson et al., 2010), and the organization of behavioral responses (Medford and Critchley, 2010). Moreover, the SN can initiate cognitive functions by sending control signals to other large-scale networks that are associated with the allocation of attention and working memory resources (Menon, 2011).
Meanwhile, the amygdala and the substantia nigra/ventral tegmental area (SNc/VTA), two subcortical structures of the SN, are involved in detecting emotional and reward saliency (Seeley et al., 2007; Menon, 2011).

In summary, this study aimed to examine the role of the synchronizing activity in the FPN and/or SN in the motivation-based facilitation of proactive control. To this end, we hypothesized that these two networks would show the following features: (1) FPN/SN may be engaged in the proactive control task (i.e., AX-Continuous Performance Task, AX-CPT) whereby the activity of these two network is associated with the task phase and task performance; (2) task engagement of FPN/SN may be associated with proactive control index which was measured by the relative performance of $\mathrm{BX}$ and $\mathrm{AY}$ trials; and (3) reward may modulate the association between FPN/SN engagement and proactive control. Specifically, we assumed that reward might promote the relationship between FPN/SN engagement and proactive control. Moreover, previous studies have suggested that the effect of reward motivation on cognitive control can be reflected in the interaction between two large-scale brain networks. These include one brain network representing the reward value and another performing specific cognitive functions (Botvinick and Braver, 2015). Based on the proposed functioning of SN (i.e., detection of reward saliency and translation of control signals to other brain networks) and FPN (i.e., motivation-based enhancement of proactive control), we propose that $\mathrm{SN}$ activity may represent the reward value and signal FPN to implement specific control functions. We therefore hypothesized that: (1) reward may modulate the relationship between SN and FPN activity (or connectivity between these two networks), making them more correlated in the reward condition; and (2) FPN activation may mediate the positive relationship between $\mathrm{SN}$ activity and proactive control. That is, $\mathrm{SN}$ activity may enhance the activation of the FPN, which in turn promotes proactive control.

\section{MATERIALS AND METHODS}

\section{Participants}

Twenty-four right-handed, healthy young adults participated in this study. All of them had normal or corrected-to-normal vision, and none of them had a history of neurological or psychiatric illness. Two volunteers were excluded, as they did not complete the testing. In addition, another two subjects were excluded as their overall head motion was above $2 \mathrm{~mm}$ in translation or $2^{\circ}$ in rotation during scanning. In total, 20 subjects were included in the subsequent analysis, which consists of nine females (mean $=21.7$ years, $\mathrm{SD}=1.8$ ) and 11 males (mean $=21.6$ years, SD =1.4). The study was approved by the Southwest University Brain Imaging Center Institutional Review Board. All subjects gave written informed consent in accordance with the Declaration of Helsinki.

\section{Task and Procedure}

Participants performed the reward version of the AX-CPT in a block design. The AX-CPT has been widely used to investigate 
the dynamic changes between proactive and reactive control (Braver, 2012). In the classical AX-CPT, participants need to respond to a probe $(\mathrm{X} / \mathrm{Y})$ which follows a cue $(\mathrm{A} / \mathrm{B})$. " $\mathrm{B}$ " represents any non-A letters and " $Y$ " stands for any non-X letters. Only the A-cue followed by the $\mathrm{X}$-probe requires a target response, whereas all other combinations (A-Y, B-X, $\mathrm{B}-\mathrm{Y})$ require a non-target response. In this study, the cue letter was either $\mathrm{A}$ or $\mathrm{B}$, and the probe letter was either $\mathrm{X}$ or Y. Participants were asked to respond to the target (AX combination) with the right index finger, and respond to the non-target with the left index finger (AY, BX, BY). Because the A-X combination occurs at a high frequency (70\%), both the $\mathrm{A}$-cue and the X-probe could produce high target response bias. However, the inconsistent response tendency between the cue and the probe in the $\mathrm{A}-\mathrm{Y}$ and the $\mathrm{B}-\mathrm{X}$ combinations would cause interference, participants thus need to switch between proactive and reactive control strategies to achieve better task performance. Specifically, a proactive control strategy helps resolve the conflict in the $\mathrm{B}-\mathrm{X}$ combination, but a reactive control strategy is useful for resolving the interference in the A-Y combination (Locke and Braver, 2008; Chatham et al., 2009). Thus, task performance in the AY and BX trials can be considered as an index of the reactive and proactive control strategy respectively. Here, we calculated proactive control index for both RT and error rate using the behavioral shift index (BSI: $[\mathrm{AY}-\mathrm{BX}] /[\mathrm{AY}+\mathrm{BX}]$ ), which measures to what extent subjects tend to adopt the proactive control strategy (Paxton et al., 2006, 2008; Chiew and Braver, 2014). A smaller value of this index means less proactive and more reactive control, while a larger value means more proactive and less reactive control (Lamm et al., 2013; Zhang et al., 2015; Maraver et al., 2016).

In the reward context, participants were informed that they would be rewarded for their quick and correct response if the prime stimulus of that trial is " $\$ \$ \$$," but not if the prime is “\#\#." In the baseline context, neither incentives nor the meaning of the motivation cues were provided. Thus, the feedback " +50 " was only presented in the reward context which means that participants would receive 50 cents, and “+--" was presented in both the reward and baseline context indicating that no reward was provided. There were two runs, i.e., a baseline and a reward run in the task, and each run has three blocks. The baseline run was followed by the reward run. The sequence was fixed because reward was only provided if the response in the reward block was correct and faster than the average reaction time (RT) in the baseline block (Locke and Braver, 2008; Chiew and Braver, 2013). Each block included 40 trials, with 28 AX trials (70\%), 4 AY trials (10\%), 4 BX trials (10\%) and 4 BY trials (10\%). These trials were presented in a pseudorandom order and each block lasted for $9 \mathrm{~min}$. A 10 -s rest was set at the beginning of the experiment, and the imaging data in this period were abandoned so that the scanner could reach a steady state. One practice block was conducted before the actual testing. The actual task started if the accuracy in the practice block reached at least $90 \%$.

An illustration of the task is provided in Figure 1. The letters of the cue and target stimuli were presented at the center of the screen in sequence in 36-point size, Times New Roman font. A random jitter (fixation, $2 \mathrm{~s}, 4 \mathrm{~s}$, or $6 \mathrm{~s}$ ) was set for the inter-trial interval (ITI). Then a reward symbol (“\$\$” or "\#\#\#”) and the following mask were presented for $300 \mathrm{~ms}$. Next, a random jitter fixation screen $(300 \mathrm{~ms}, 600 \mathrm{~ms}$, or $900 \mathrm{~ms}$ ) was shown before the cue screen $(300 \mathrm{~ms})$, followed by a delay period $(4,700 \mathrm{~ms})$, a target screen $(300 \mathrm{~ms})$ and a blank screen for response $(1,700 \mathrm{~ms})$. The timing for response was fixed to $2,000 \mathrm{~ms}$ and not terminated by a response. Then, a feedback with the following mask screen $(300 \mathrm{~ms})$ were displayed. In the end of each trial, a reminder for the next trial "Next!" was presented for $300 \mathrm{~ms}$.

\section{fMRI Data Collection}

All functional magnetic resonance imaging (fMRI) were collected on a Siemens 3T Trio scanner (Siemens Medical Systems, Erlangen, Germany). A foam pad was used to minimize the subjects' head motion. fMRI images were acquired by using the gradient-echo echo planar imaging (GRE-EPI) sequence: $\mathrm{TR} / \mathrm{TE}=2,000 \mathrm{~ms} / 30 \mathrm{~ms}$; flip angle $=90^{\circ}$; resolution matrix $=64 \times 64 ; \mathrm{FOV}=220 \times 220 \mathrm{~mm}^{2}$; thickness $=4 \mathrm{~mm}$; and acquisition voxel size $=3.4 \times 3.4 \times 4 \mathrm{~mm}^{3}$. We used 32 slices to cover the entire brain.

\section{Data Preprocessing}

fMRI data were preprocessed using statistical parametric mapping 8 (SPM8, Welcome Trust Centre for Neuroimaging, London, $\mathrm{UK}^{1}$ ) implemented on a MATLAB 2009b (Math Works, Natick, MA, USA) platform. For each block, the first 5 functional volumes were discarded. The remaining scans were slice-time corrected and subsequently realigned to the first image to correct for head motion. Subsequently, all realigned images were spatially normalized to the Montreal Neurological Institute (MNI) template and resampled into $3 \times 3 \times 3 \mathrm{~mm}^{3}$. Thereafter, the images were smoothed with a $6 \mathrm{~mm}$ full-width at half-maximum (FWHM) Gaussian kernel to increase the signalto-noise ratio (SNR).

\section{Behavioral Data Analysis}

We first calculated the relative proactive control index for both RT and error rate using the formula $[(\mathrm{AY}-\mathrm{BX}) /(\mathrm{AY}+$ BX)] (Paxton et al., 2006, 2008; Chiew and Braver, 2014). This proactive index is a standardized measure that ranges from -1 to +1 . There were three levels of incentive in this study, namely, the baseline trials, non-incentive trials in the reward context and incentive trials in the reward context. To examine the reward effect on proactive control, baseline trials and incentive trials in the reward block were examined with paired sample $t$-tests. The ANOVA analysis between reward prime (\#\#\# vs. \$\$) and block (baseline vs. reward block) was not presented here as we were not interested in the main or the interaction effect between these two variable. Moreover, we did not find any significant result of the main or the interaction effect between reward prime and block.

\footnotetext{
${ }^{1}$ http://www.fil.ion.ucl.ac.uk/spm
} 


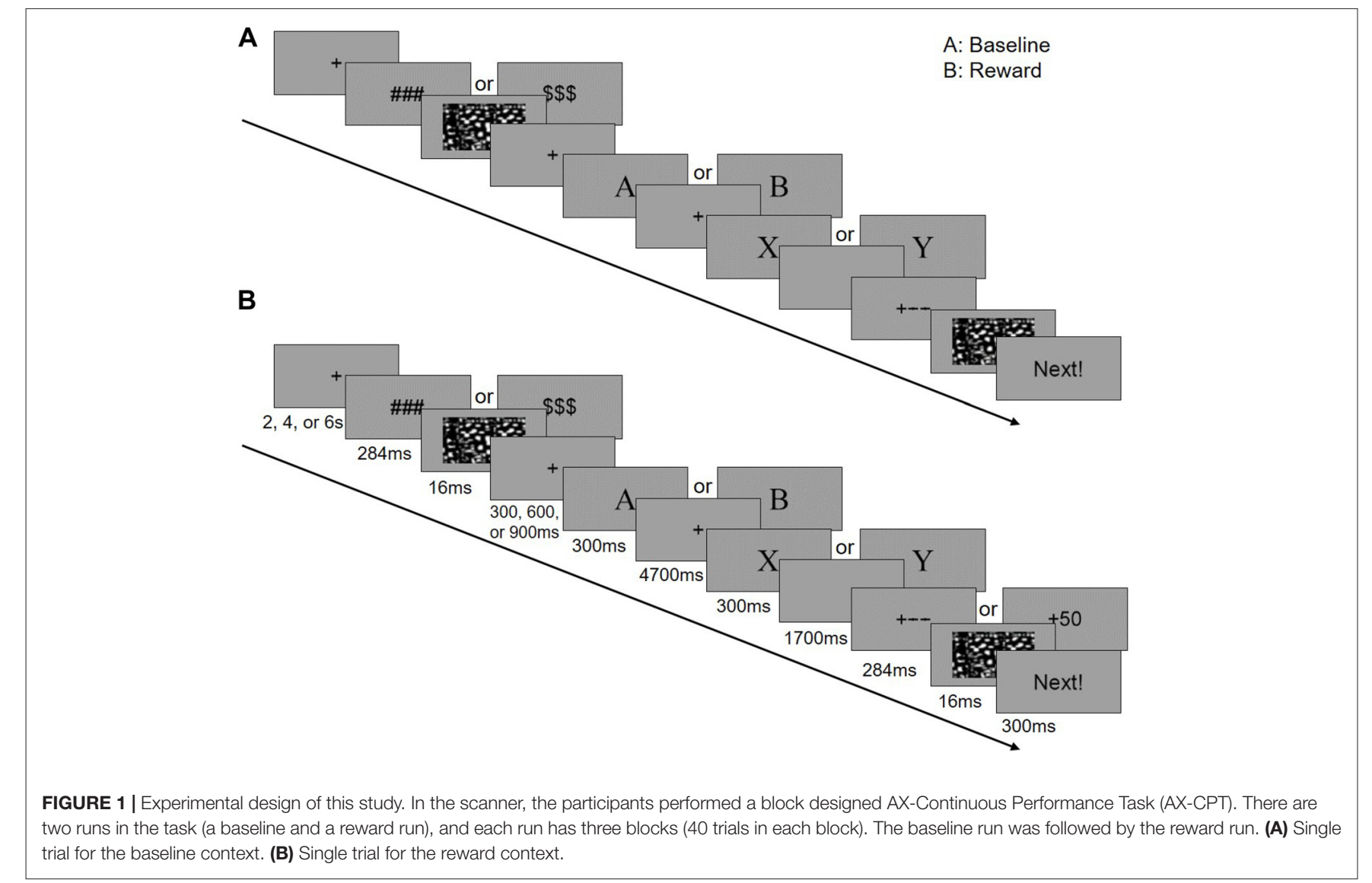

\section{Independent Component Analysis of fMRI Data}

Functional imaging data can be decomposed to brain networks using independent component analysis (ICA), which has been increasingly applied to task-fMRI data (Xu et al., 2013a, 2016; Yip et al., 2018). ICA is a data-driven method to characterize the temporal coherence of neural activities and functional integration of neural networks (Meda et al., 2012; Smith et al., 2012). Meanwhile, cognitive task performance involves the recruitment of multiple brain regions, it is plausible that reward may modulate the functional integration between these various task related networks, rather than a single region. Moreover, ICA is sensitive to neural activity patterns that may not be detected by traditional whole-brain analysis based on general linear model (GLM), which has been suggested to be able to provide supplementary information to GLM-based methods (Xu et al., 2016; Yip et al., 2018). Furthermore, in this study, we are interested in understanding the functional synchronization (connectivity) of the brain regions or networks, which provides a context for ICA to fit in.

ICA was performed using the MATLAB platform group ICA toolbox (GIFT2 ${ }^{2}$, version 3.0a), which can identify spatially

\footnotetext{
${ }^{2}$ http://icatb.sourceforge.net
}

independent and temporally coherent networks. Preprocessed fMRI data were submitted to principal component analysis to reduce the dimensions for each subject. Then group spatial ICA was conducted with the Infomax algorithm (Bell and Sejnowski, 1995), and the number of component in our data was estimated to be 24 using minimum description length criteria in the software (Li et al., 2007). Group spatial ICA generates spatial maps and time series of bold signals for each component. The spatial maps characterize the brain regions included in the network, with values in these maps representing the temporal synchronization between the time series of a given voxel and a given network, reflecting how strongly each voxel is coupled with a given network (Allen et al., 2012). Each subject's time courses and spatial maps were then back-reconstructed by using the data from the data reduction procedure. In total, 24 spatial maps and related time series were produced for each participant. We can carry out group-level random effect hypothesis tests to compare individual differences or condition differences in each spatial map or component time series. The analysis of spatial maps allows us to compare the group difference in the intensity of functional connectivity, while the analysis of the temporal process information enables us to judge whether the participants engaged in the fMRI task or not (Meda et al., 2009).

In the context of task-fMRI, some components may reflect networks that are helpful in performing tasks, while other 
components may represent unrelated intrinsically connected networks or spatiotemporal sources of noise (Hallquist et al., 2018). To identify the task-related network, we performed regression analysis between component time series and stimulus events convolved with a canonical hemodynamic response function (HRF), which is usually applied in ICA of task-fMRI data (e.g., Zhang and Li, 2012). Similar to conventional voxel wise GLM analyses, the resulting regression coefficients (beta values) represent the influence (correlation) of the stimulus on BOLD activity (Kim et al., 2009). The main difference between ICA and single voxel analysis is that component time courses represent a group of temporally coherent activities in functional coupling regions. Therefore, like voxel wise GLM regression coefficient, the correlation between component time courses and task phase reflects both the intensity of task-related network activity and the temporal accordance of the network to stimulus presentation (Hallquist et al., 2018). Thus, an increase or decrease in regression coefficient (beta values) in one condition relative to another represents an increase or decrease in the task-related activity of the corresponding network (Xu et al., 2013b).

\section{Identification of the Task Related Network}

We first examined if the FPN/SN activation is associated with the task phase of the AX-CPT as well as the task performance. Three separate boxcar regressors were first defined by a train of cue onsets with a duration of $6 \mathrm{~s}$ (form cue onset to the blank screen waiting for response to include 3TR), representing all trials (including AX, AY, BX, BY), AY trials and BX trials, respectively (Figure 2). These regressors were then convolved with a canonical HRF. Temporal regression was then performed between the time courses of the 24 components and three task regressors. By doing this we can estimate the association (beta weights) between the component time series and the three task regressors. The beta values represent the degree of synchrony between the components' time courses and the task phase, which indicates whether the network was engaged during this task (Meda et al., 2009). An increase or decrease in beta values in one condition relative to another represents an increase or decrease in the task-related activity of the corresponding network (Xu et al., 2013b). We first visually checked the most correlated network, than we performed a spatial correlation between a predefined SN mask ${ }^{3}$ (Shirer et al., 2012) and the identified components. This template included the main SN regions such as the bilateral anterior insula (AIns) and the dorsal anterior cingulate (dACC; Seeley et al., 2007). To further examine whether or not FPN and $\mathrm{SN}$ are involved in the task, we calculated the correlation between network activity (beta values) and behavioral performance for both RT and ACC, separately.

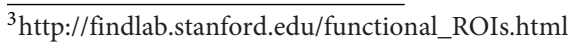

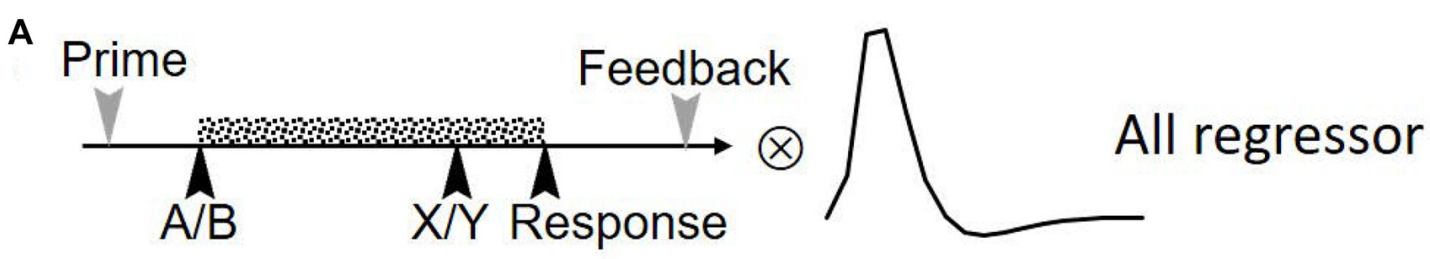

B

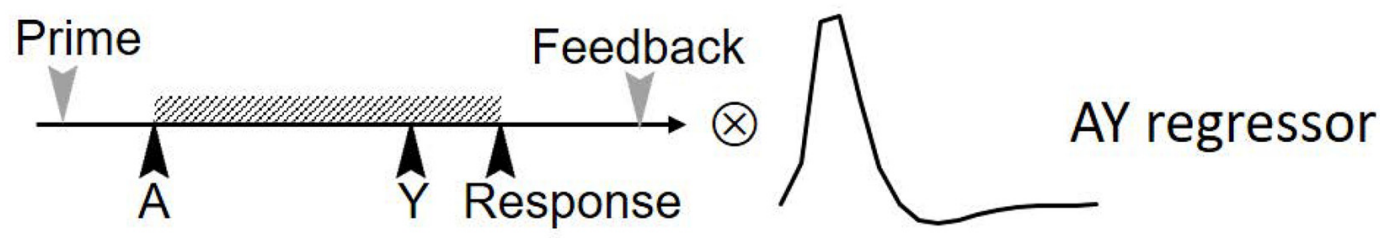

C

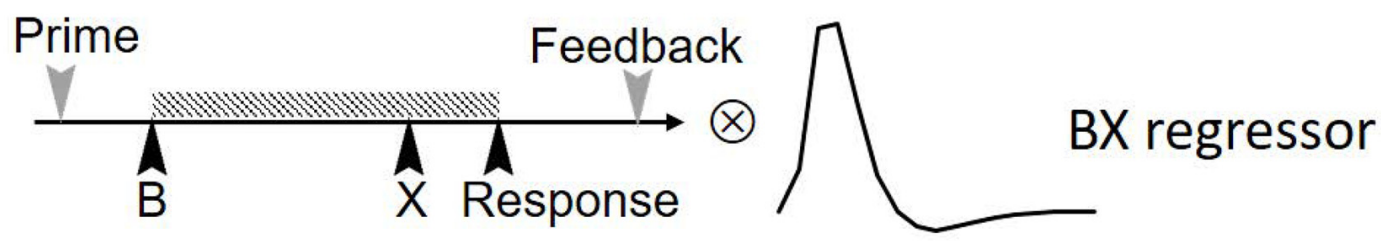

FIGURE 2 | Three regression factors for the task in the study. Three independent boxcar-shaped regression factors were set up. These three sequences represent the regression factors for (A) all trials (including AX, AY, BX, BY), (B) AY trials and (C) BX trials. The three sequences were convolved with statistical parametric mapping's (SPM's) hemodynamic response function (HRF) to form the final task sequence. 


\section{The Association Among Reward, AY/BX Engagement of the Salience Network and Proactive Control}

Since we found SN but not FPN is involved in the task, the following analysis was only performed on the SN. We first calculated the association between AY/BX engagement of the SN and proactive control, with the assumption that $\mathrm{SN}$ engagement in $\mathrm{AY} / \mathrm{BX}$ trials may predict the behavioral proactive control index. Considering that better performance on BX trials indicates proactive control, and that better performance on AY trials indicates reactive control (Chatham et al., 2009), higher level of $\mathrm{BX}-\mathrm{AY}$ engagement would be associated with better proactive control.

Subsequently, we explored whether reward could modulate the association between AY/BX engagement of the SN and proactive control. To this end, the correlation coefficients between BX-AY engagement of the $\mathrm{SN}$ and proactive control of the baseline and reward condition were calculated, respectively. Then, Fisher's $r$-to- $z$ transformation was performed to increase the normality of the correlation coefficients. Finally, $z$ values of correlation coefficients were subjected to significant tests.

\section{The Correlation Among Reward, Trial Types and AY/BX Engagement of the Salience Network}

We further explored why reward might modulate the association between AY/BX engagement of the SN and proactive control. As the relationship between BX-AY engagement of the $\mathrm{SN}$ and proactive control might be greater in the reward condition, we assumed that reward might enhance task engagement of SN proactively. In other words, compared to baseline, reward might enhance BX engagement more than AY engagement of the SN. This proactive neural network engagement may support the behavioral proactive performance. We thus submitted the $\mathrm{AY} / \mathrm{BX}$ engagements to repeated measure ANOVA with trial types (AY vs. BX) and reward (reward vs. baseline) as withinsubject factors to determine if the interaction between trial types and reward is significant.

\section{RESULTS}

\section{Behavioral Results}

To validate the effect that reward can modulate proactive control, a paired sample $t$-test was performed to compare the proactive control of baseline trials with incentive trials in reward block. Results revealed that incentive trials in reward block showed higher proactive control for both RT $\left(t_{(19)}=2.44, p=0.02\right)$ and error rate $\left(t_{(19)}=3.07, p=0.01\right.$; Figure 3$)$. This result is consistent with previous studies showing that reward can facilitate proactive control (Locke and Braver, 2008; Jimura et al., 2010; but see Boehler et al., 2014).

\section{The Salience Network Is Engaged in the AX-CPT}

Visual inspection suggested that the most positively correlated network with the task was SN $\left(t_{(19)}=10.73, p=1.67 \times 10^{-9}\right)$. Regions, $T$ values, and MNI coordinates of this network were listed in Table 1. Spatial correlation results confirmed

TABLE 1 | Regions of the salience network (SN) that are correlated with the task phase of AX-CPT.

\begin{tabular}{lcrrrr}
\hline Regions & Voxels (n) & $\boldsymbol{T}$ & \multicolumn{3}{c}{ MNI coordinates } \\
\cline { 4 - 6 } & & & $\boldsymbol{x}$ & $\boldsymbol{y}$ & $\boldsymbol{z}$ \\
\hline SMA/dACC & 721 & 10.8 & 9 & 8 & 49 \\
L. Alns & 137 & 8.1 & -30 & 23 & 4 \\
R. Alns & 170 & 10.8 & 36 & 17 & 4 \\
L. MFG & 121 & 6.8 & -30 & 50 & 19 \\
R. MFG & 103 & 5.8 & 30 & 50 & 25
\end{tabular}

Corrected at $p<0.01$. AX-CPT, AX-Continuous Performance Task; SMA, supplementary motor area; $d A C C$, dorsal anterior cingulate; Alns, anterior insula; MFG, middle frontal gyrus; L, Left; $R$, right.
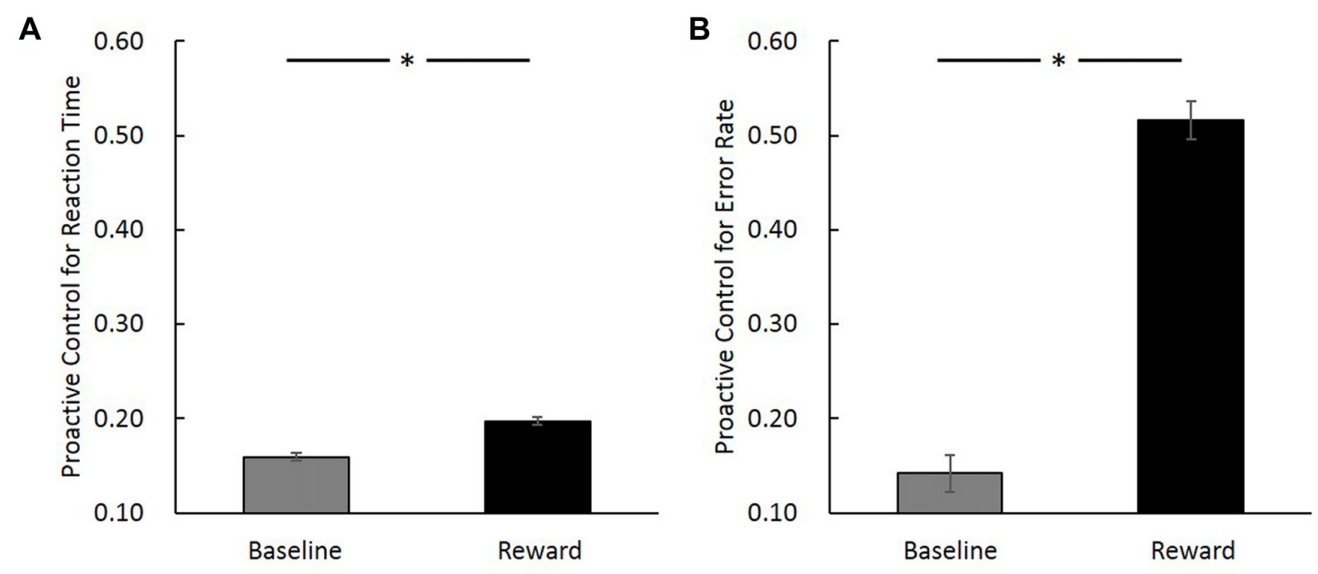

FIGURE 3 | Proactive control index ([AY - BX]/[AY + BX]) in baseline and reward block (reward trials in the incentive block) for reaction time (RT; $\mathbf{A})$ and error rate (B). The horizontal axis denotes different conditions (baseline vs. reward) and the vertical axis represents the magnitude of proactive control (error bars denote standard errors). ${ }^{*}$ Indicates $p<0.05$ 
the relationship in which this component showed the highest positive spatial correlation with the SN template (0.57). We also submitted a FPN template ${ }^{4}$ for spatial correlation and identified the component that showed the highest spatial correlation (0.45). However, this component was not correlated with the task $(p>0.1)$.

Among the 24 components, we found that SN activity is associated with the AX-CPT task. One-sample $t$-tests were applied to the beta values of the SN to verify that the $\mathrm{SN}$ time series was significantly $\left(t_{(19)}=10.73, p=1.67 \times 10^{-9}\right)$ associated with the task course and was thus task-engaged (Figure 4). To be more confirmative, the correlations between the task engagement of the $\mathrm{SN}$ and the behavioral data (RT and error rate) were examined. A more task-engaged SN was associated with a faster RT $\left(r_{(18)}=-0.55, p=0.02\right.$; Figure 5) but not for error rate $\left(r_{(18)}=-0.36, p=0.12\right)$, which indicates that the $\mathrm{SN}$ is involved in the AX-CPT task. The deeper SN engaged in the task, the faster RT would show. Meanwhile, the activity of FPN was not correlated with either RT $\left(r_{(18)}=-0.07, p=0.078\right)$ or error rate $\left(r_{(18)}=-0.004, p=0.99\right)$. To avoid possible false positive results, multiple comparison correction was applied to our results by setting false discovery rate (FDR) to $<0.05$ (Benjamini and Hochberg, 1995; Benjamini and Yekutieli, 2001).

\section{Reward Enhances the Correlation Between AY/BX Engagement of the Salience Network, and Proactive Control}

The correlation between BX-AY engagement of the $\mathrm{SN}$ and proactive control index for both $\mathrm{RT}$ and error rate were calculated, respectively. We found that BX-AY engagement of the $\mathrm{SN}$, which may reflect the level that $\mathrm{SN}$ is proactively engaged, is positively associated with proactive control index for RT $\left(r_{(18)}=0.43, p=0.056\right)$, but not for error rate $\left(r_{(18)}=0.24, p=0.32\right)$. Greater difference between BX and

$\overline{{ }^{4} \text { http://findlab.stanford.edu/functional_ROIs.html }}$
AY engagement was associated with better proactive control. Further analysis revealed that this positive correlation was only present in the reward condition $\left(r_{(18)}=0.51, p=0.02\right)$, but not for the baseline $\left(r_{(18)}=0.08, p=0.75\right)$. Moreover, the difference between these two correlation coefficients was significant $\left(Z=5.9, p=1.79 \times 10^{-9}\right.$; Figure 6A). Thus, reward enhanced the correlation between $\mathrm{BX}-\mathrm{AY}$ engagement of the $\mathrm{SN}$ and proactive control, making them positively correlated.

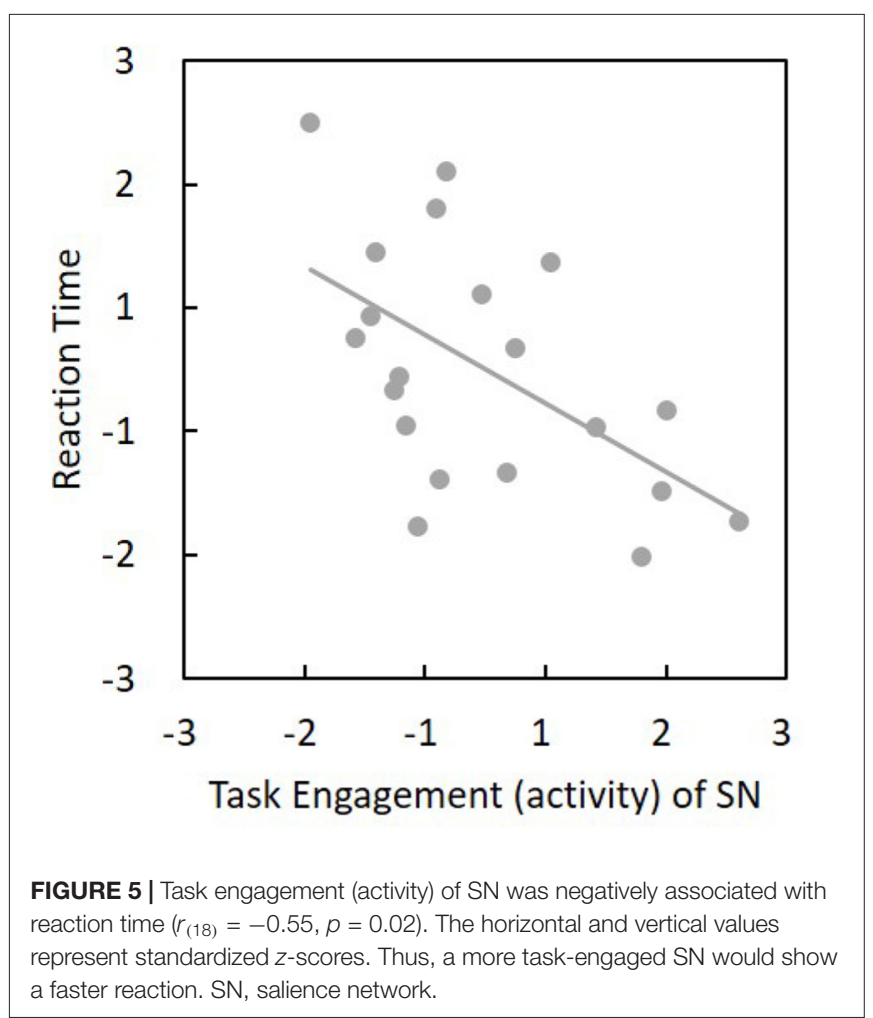

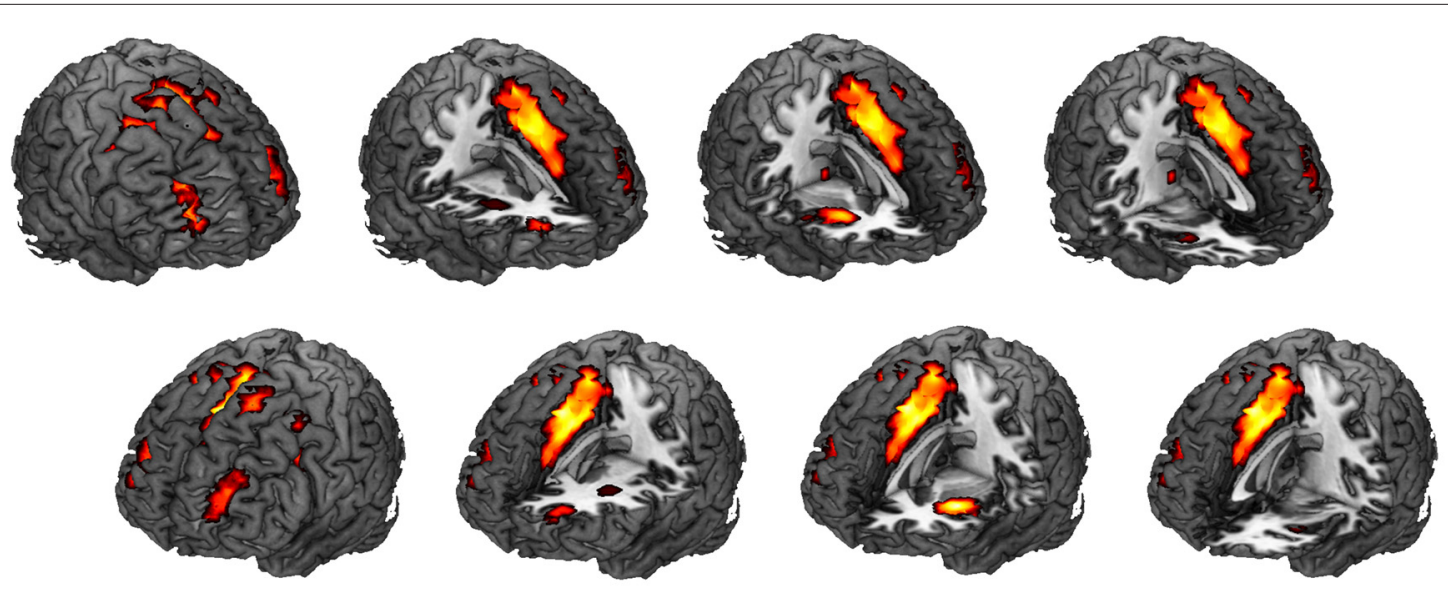

FIGURE 4 | Spatial map for the component that was most correlated with the task. One sample $t$-test was performed for this spatial map (corrected for multiple comparisons with the FDR, $p<0.01$ ). See Table 1 for specific regions, $T$ values and montreal neurological institute (MNI) coordinates. This component showed the highest positive spatial correlation (0.57) with an SN template. FDR, false discovery rate; SN, salience network. 

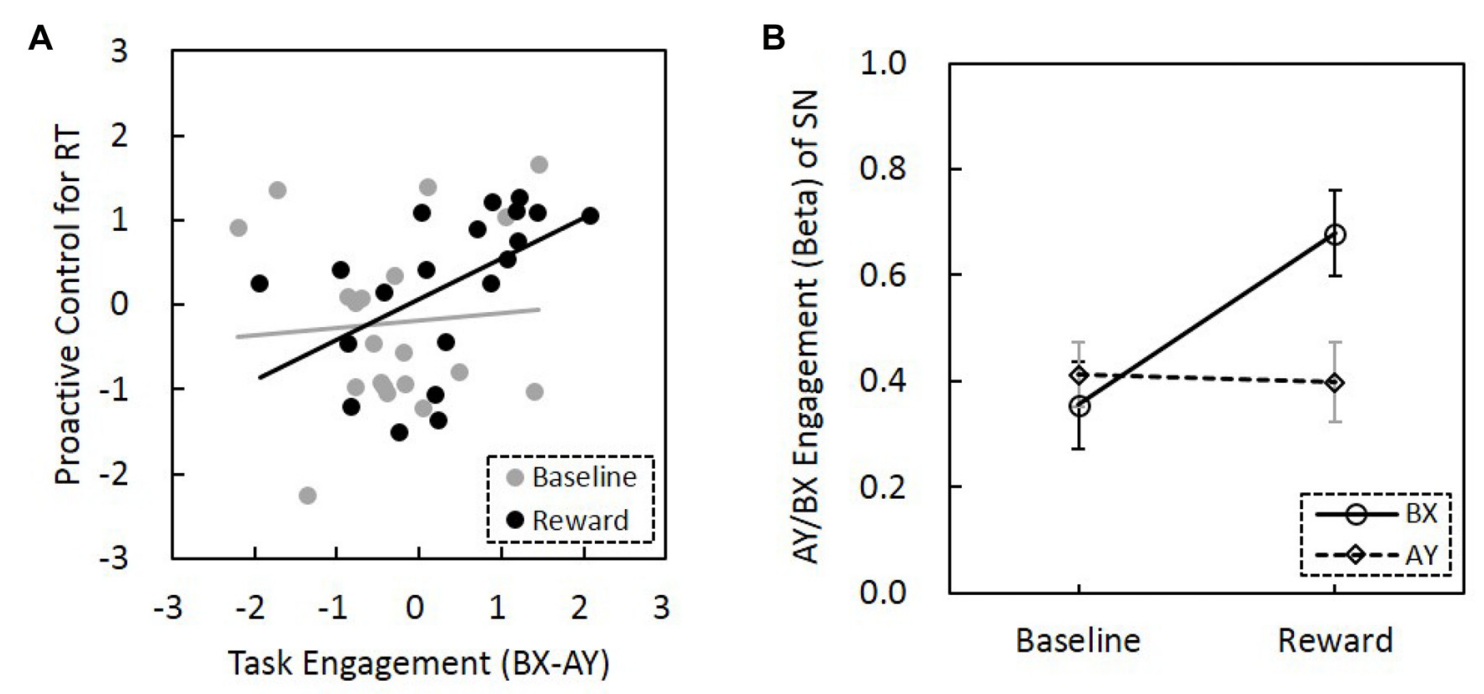

FIGURE 6 | Relationship among reward motivation, task engagement of SN and proactive control. (A) Compared to the baseline, reward motivation increased the association between task engagement (activity) of SN (BX-AY) and proactive control. Before the comparison, Fisher's r-to-z transformation was performed to increase the normality of the correlation coefficients, and $z$ values of correlation coefficients were subjected to paired sample $t$-test (baseline vs. reward). The horizontal and vertical values represent standardized z-scores. (B) The interaction between reward and trial types was significant, suggesting reward differentially influenced the SN engagement for AY and BX trials (error bars denote standard errors). SN, salience network.

\section{Reward Modulate the AY/BX Engagement of the Salience Network}

The ANOVA for the AY/BX engagement of the SN found a significant main effect of reward $\left(F_{(1,19)}=5.66, p=0.021\right.$; Figure 6B). SN engagement was higher for the AY and BX trials in the reward condition than in the no-reward condition. The effect of trial type was not significant $\left(F_{(1,19)}=2.5, p=0.12\right)$. Importantly, the interaction between reward and trial types was significant $\left(F_{(1,19)}=4.61, p=0.036\right)$, that is, reward can promote significantly greater task engagement of $\mathrm{SN}$ in trials that need proactive control (BX trials) than trials that need reactive control (AY trials). Thus, reward might enhance task engagement of SN proactively, and this proactive neural network engagement might support the behavioral proactive performance.

\section{DISCUSSION}

The present study investigated the relationship among reward, task engagement of the $\mathrm{SN}$, and proactive control. Consistent with previous studies (Locke and Braver, 2008; Jimura et al., 2010), our results demonstrated that reward motivation could enhance the proactive mode of cognitive control. We further found that the SN is involved in the AX-CPT task, in which the time series of $\mathrm{SN}$ is positively correlated with task regressor, and that the task engagement of $\mathrm{SN}$ is positively associated with behavioral performance. Interestingly, our data demonstrated that reward could modulate the association between the $\mathrm{SN}$ engagement and proactive control whereby the SN engagement is positively associated with proactive control particularly in the reward condition. Furthermore, we found a significant interaction effect between reward and trial types, indicating that reward enhances greater task engagement of $\mathrm{SN}$ in trials that need proactive control (BX trials) than trials that need reactive control (AY trials). However, it is possible that the different responses between A and B cue may account for some of the results. Therefore, it is purposed that the SN network may be more engaged in B cue compared to A cue, suggesting increased utilization of contextual cues (proactive control) as $B$ cues are $100 \%$ valid for preparing a non-target response (Braem et al., 2014; Fröber and Dreisbach, 2014; Hefer and Dreisbach, 2017).

We explored the control mode shift (tendency) toward relative proactive control, as indicated by the BSI ([AY $\mathrm{BX}] /[\mathrm{AY}+\mathrm{BX}])$. A smaller value of this index means less proactive and more reactive control, while a larger value means more proactive and less reactive control (Lamm et al., 2013; Zhang et al., 2015; Maraver et al., 2016). In this regard, we assume that reward may decrease reactive control, since task performance in AY and BX trials can be considered as an index of the reactive and proactive control strategy, respectively (Braver et al., 2009; Polizzotto et al., 2018). Therefore, higher value of the BSI in the reward condition would also imply relatively lower reactive control in $\mathrm{AX}-\mathrm{CPT}$. It is reported that reward can enhance the task coding (Etzel et al., 2015), increase the maintenance of goal-relevant information (Zedelius et al., 2011), and promote cognitive stability (Fröber and Dreisbach, 2016; Hefer and Dreisbach, 2016, 2017; Fröber et al., 2018). In the reward condition of $\mathrm{AX}-\mathrm{CPT}$, the coding (representation) of $\mathrm{A} / \mathrm{B}$-cue and the corresponding response tendencies may be increased, which was also more stably maintained in the following period until response was made. Therefore, the A-cue would induce and stably maintain a stronger target response tendency, which would decrease the flexibility and 
detrimental for AY trials (non-target). While the B-cue would induce and stably maintain a stronger non-target response tendency, which is beneficial for BX trials (non-target; Hefer and Dreisbach, 2017; Yee and Braver, 2018). Thus, reward may increase proactive control and decrease reactive control in $\mathrm{AX}-\mathrm{CPT}$.

In this study, we found that the SN activity is associated with the AX-CPT task. SN is widely involved in cognitive control processes, e.g., conflict monitoring (Kerns et al., 2004), salience processing (Seeley et al., 2007), interference resolution (Nee et al., 2007), the maintenance and implementation of task sets (Dosenbach et al., 2006; Nelson et al., 2010), and the organization of behavioral responses (Medford and Critchley, 2010). Individuals need to flexibly adjust the proactive and reactive control strategies to solve the conflict in $\mathrm{AY}$ and $\mathrm{BX}$ trials in this protocol. Moreover, each trial type of AY, BX and BY only covers $10 \%$ of all trials. Thus, these trials may be considered as salience or oddball stimuli. In addition, the delay period between the cue and the probe requires the maintenance of goal-related information in working memory. In this regard, it is not surprising that the $\mathrm{SN}$ is engaged in AX-CPT, which could measure relative proactive control. The higher activation of the $\mathrm{SN}$ in the task, the better the task performance.

Our data demonstrated that reward could modulate the association between the SN engagement and proactive control. Previous studies have shown that SN is involved in rewardbased decision making (Kennerley et al., 2011; Botvinick and Braver, 2015). Moreover, dACC, a core node of SN, plays a key role in the association between reward and cognitive control. Specifically, the expected values of control are suggested to be computed in the dACC, which then selects appropriate control functions based on the expected values. These cognitive controls are subsequently implemented in the sub-regions of the lateral PFC (Shenhav et al., 2013, 2016). Moreover, the SN is structurally connected with the subcortical and limbic structures such as the amygdala and SNc/VTA, which are involved in the processing of reward and motivation (Menon and Uddin, 2010; García-García et al., 2013). Importantly, SN includes regions (e.g., dACC and AIns) that are responsive for interoceptiveautonomic, conflict monitoring and reward-processing (Seeley et al., 2007). Information from these regions may thus be integrated by $\mathrm{SN}$ to relay the signal to other brain regions or organize a response. Therefore, reward motivation in this study may result in enhanced reward coding in the $\mathrm{SN}$, which is associated with relatively more proactive control.

Importantly, our data demonstrated that reward could modulate the AY/BX engagement of the SN proactively, resulting in greater improvement of BX engagement than $\mathrm{AY}$ engagement. This neural modulation of the SN activity may account for the behavioral proactive performance after reward, since both ACC and the dopamine (DA; a neurotransmitter that is important for reward, motivation and cognitive control function) system can link reward with cognitive control (Botvinick and Braver, 2015; Westbrook and Braver, 2016). Moreover, phasic activity of DA neurons was found in response to reward cues (Schultz, 1998), while tonic DA release was associated with sustained motivational behaviors (Howe et al., 2013). In fact, the phasic and tonic DA signals not only support the processing of reward and motivational information (Niv, 2007), but are also involved in the modulation of proactive control (Cohen et al., 2002). Thus, it may be the case that reward increases the striatal DA release, which then modulates task engagement of the SN and promotes the persistence cognitive control (Westbrook and Braver, 2016).

Surprisingly, we found that the FPN is not involved in the task. It seems counter-intuitive since lateral PFC as one of the core regions of FPN has been commonly activated in motivation-cognitive control interaction studies (e.g., Bahlmann et al., 2015). However, it is possible that a single region (lateral PFC) may not be sufficient to represent FPN, as part of the lateral PFC may also belong to SN. The SN identified in this study includes portion of the lateral PFC, though the core regions of SN were AIns and dACC (Figure 4 and Table 1). Therefore, SN but not FPN in our result may be compatible with previous studies. It has been suggested that BOLD signal in the SN comes earlier than the FPN, and that activity of the SN may modulate FPN activity. For instance, a number of studies suggested that the SN plays a causal role in the switching between the FPN and the default mode network (e.g., Sridharan et al., 2008; Goulden et al., 2014). The dACC as a key node in SN was also found to signal the FPN node (dorsolateral PFC) for top-down control (Botvinick et al., 2001; Kerns et al., 2004; Liston et al., 2006). Therefore, SN activation may be necessary to initiate the functioning of the FPN. In line with this idea, prior studies have indicated that the AIns (one core region of the $\mathrm{SN}$ ) contributes to the generation of control signals that are critical for the "stable maintenance of task mode and strategy" (Dosenbach et al., 2007). Menon and Uddin (2010) further assumed that the AIns is involved in the detection of salient stimuli. When a salient stimulus is detected, the AIns initiates control signals that are then sustained by the ACC and the lateral PFC. Similarly, it is proposed that cognitive control includes three major components, namely, monitoring, specification and regulation (Shenhav et al., 2013, 2016). The dACC monitors the current circumstances and notifies the lateral PFC which task should be undertaken, while the lateral PFC has the capacity to conduct or regulate lower-level information processing (Shenhav et al., 2013, 2016). It is therefore possible that the SN activation in our data may reflect the process of salience monitoring and task specification, while the lateral PFC activity in previous studies is more likely to be associated with the regulation component of cognitive control.

There are other possibilities with regard to the findings of the FPN in this study. We examined this issue from a network perspective, which is different from previous studies that investigated the activations of specific brain regions. Furthermore, ICA was employed in this study to dissociate multiple components. Although most component maps from group ICAs are stable, there are still subtle differences in spatial extent of these components each time ICA is applied (Beall and Lowe, 2010). Therefore, the FPN identified by ICA in this study may be subject to the random covariate. Moreover, the trial number is relatively small, resulting to a relatively low SNR. Whether FPN as a network involved in the incentive 
enhancement of proactive control therefore remains to be established in future studies.

\section{LIMITATION}

As the order of baseline and reward blocks is fixed, it is possible that the block-based results are confounded by the practice effect. However, this is not necessarily the case here since previous studies did not find practice effect in block-based reward studies (Chiew and Braver, 2013). Moreover, we performed a control analysis and did not find any practice effect in our study. Specifically, a series of paired sample $t$-tests were performed including the comparison between the first and the second block (40 trials) in baseline (RT: $t_{(19)}=0.53, p=0.6$; error rate: $\left.t_{(19)}=0.94, p=0.36\right)$, between the first and the last block in baseline $\left(\mathrm{RT}: t_{(19)}=-0.29, p=0.78\right.$; error rate: $t_{(19)}=1.29$, $p=0.21$ ), and between the first half and the last half of trials in the baseline $\left(\mathrm{RT}: t_{(19)}=0.09, p=0.93\right.$; error rate: $t_{(19)}=1.25$, $p=0.23$ ). Nevertheless, this issue should be further examined in future studies with careful experimental designs.

Note that previous studies commonly explored the reward effect by comparing non-incentive trials in the reward block to baseline trials (sustained effect), and incentive trials in the reward block to non-incentive trials in the reward block (transient; Chiew and Braver, 2013, 2014). In this study, we compared incentive trials in the reward block and baseline trials because the aim of this study was not to dissociate the sustained (blockbased) and the transient (trial-based) reward effect. We were interested in the reward facilitation effect of proactive control, and the incentive trials in the reward block have the highest motivation level. Moreover, the number of each trial type was not balanced because of the feature of this reward version AX-CPT (i.e., the number of incentive and non-incentive trials are determined by task performance). This is consistent with the previous studies (close to 1:4 for the number of non-incentive trials compared to that of the baseline trials; Chiew and Braver, 2013 , 2014). It would be inappropriate for the neural data to compute the association between network activities and the task phase, as the unbalanced trial number may elicit unstable and inaccurate results (Nasr et al., 2008; Cohen, 2017; Hall et al., 2018). Furthermore, we reanalyzed our data by comparing the reward block to the baseline block (Locke and Braver, 2008), and our results were consistent. A potentially larger trial number or an unequal number of trials for baseline and reward block (e.g.,

\section{REFERENCES}

Allen, E. A., Erhardt, E. B., Wei, Y., Eichele, T., and Calhoun, V. D. (2012). Capturing inter-subject variability with group independent component analysis of fMRI data: a simulation study. Neuroimage 59, 4141-4159. doi: 10.1016/j.neuroimage.2011.10.010

Bahlmann, J., Aarts, E., and D'esposito, M. (2015). Influence of motivation on control hierarchy in the human frontal cortex. J. Neurosci. 35, 3207-3217. doi: 10.1523/jneurosci.2389-14.2015

Beall, E. B., and Lowe, M. J. (2010). The non-separability of physiologic noise in functional connectivity MRI with spatial ICA at 3T. J. Neurosci. Methods 191, 263-276. doi: 10.1016/j.jneumeth.2010. 06.024
1:2, to achieve an approximately equal or close trial number of baseline, non-incentive and incentive trials) may be examined in future studies.

We used the same B/Y letters in our study, which may have some influence on our results. To date, AX-CPT has been modified into various versions including emotional AX-CPT (Lamm et al., 2013), No-Go AX-CPT (Gonthier et al., 2016), child-friendly version of the AX-CPT (Kamijo and Masaki, 2016), reward AX-CPT (Chiew and Braver, 2014), and etc. There is also a simplified version of the AX-CPT with four stimuli (two cue and two probe stimuli) that is similar to the task used in this study (Chatham et al., 2009). Moreover, previous studies also used the same $\mathrm{B} / \mathrm{Y}$ letters to explore reactive and proactive control (e.g., Chang et al., 2017), and the association among monetary incentive, social pressure and reactive/proactive control (e.g., Ličen et al., 2016). However, future studies employing different B/Y letters would be encouraged.

In summary, we explored the brain networks that may be involved in the reward facilitation effect of proactive control. We found that the SN was engaged in the AX-CPT task, and this engagement was positively associated with proactive control particularly in the reward condition. Meanwhile, reward could modulate the AY and BX engagement of the SN in a proactive way. It is possible that this proactive neural modulation supports the enhancement of behavioral proactive control after reward. Our finding suggest that reward may moderate the relationship between task engagement of the SN and proactive control. Findings in this study may provide insights of the neural networks supporting the reward-related promotion of proactive control.

\section{AUTHOR CONTRIBUTIONS}

AC, GX, HL and LX designed research. LX, LQ and YL performed research. LQ, XC, HL, LZ and YL analyzed data. LQ, $\mathrm{XC}, \mathrm{LZ}$ and $\mathrm{AC}$ wrote the article.

\section{FUNDING}

This work was supported by grants from the National Natural Science Foundation of China $(61431013,31771254)$ and the Fundamental Research Funds for the Central Universities (SWU1609106, SWU1709107).

Bell, A. J., and Sejnowski, T. J. (1995). An information-maximization approach to blind separation and blind deconvolution. Neural Comput. 7, 1129-1159. doi: 10.1162/neco.1995.7.6.1129

Benjamini, Y., and Hochberg, Y. (1995). Controlling the false discovery rate: a practical and powerful approach to multiple testing. J. R. Stat. Soc. Series B 57, 289-300

Benjamini, Y., and Yekutieli, D. (2001). The control of the false discovery rate in multiple testing under dependency. Ann. Stat. 29, 1165-1188. Available online at: https://www.jstor.org/stable/2674075.

Boehler, C. N., Schevernels, H., Hopf, J.-M., Stoppel, C. M., and Krebs, R. M. (2014). Reward prospect rapidly speeds up response inhibition via reactive control. Cogn. Affect. Behav. Neurosci. 14, 593-609. doi: 10.3758/s13415-0140251-5 
Botvinick, M., and Braver, T. (2015). Motivation and cognitive control: from behavior to neural mechanism. Annu. Rev. Psychol. 66, 83-113. doi: 10.1146/annurev-psych-010814-015044

Botvinick, M. M., Braver, T. S., Barch, D. M., Carter, C. S., and Cohen, J. D. (2001). Conflict monitoring and cognitive control. Psychol. Rev. 108, 624-652. doi: 10.1037/0033-295X.108.3.624

Braem, S., Hickey, C., Duthoo, W., and Notebaert, W. (2014). Reward determines the context-sensitivity of cognitive control. J. Exp. Psychol. Hum. Percept. Perform. 40, 1769-1778. doi: 10.1037/a0037554

Braver, T. S. (2012). The variable nature of cognitive control: a dual mechanisms framework. Trends Cogn. Sci. 16, 106-113. doi: 10.1016/j.tics.2011.12.010

Braver, T. S., Gray, J. R., and Burgess, G. C. (2007). "Explaining the many varieties of working memory variation: dual mechanisms of cognitive control," in Variation in Working Memory, eds A. R. A. Conway, C. Jarrold, M. J. Kane, A. Miyake and J. N. Towse (Oxford: Oxford University Press), 76-106.

Braver, T. S., Paxton, J. L., Locke, H. S., and Barch, D. M. (2009). Flexible neural mechanisms of cognitive control within human prefrontal cortex. Proc. Natl. Acad. Sci. U S A 106, 7351-7356. doi: 10.1073/pnas.0808187106

Chang, J.-H., Kuo, C.-Y., Huang, C.-L., and Lin, Y.-C. (2017). The flexible effect of mindfulness on cognitive control. Mindfulness 9, 792-800. doi: 10.1007/s12671-017-0816-9

Chatham, C. H., Frank, M. J., and Munakata, Y. (2009). Pupillometric and behavioral markers of a developmental shift in the temporal dynamics of cognitive control. Proc. Natl. Acad. Sci. U S A 106, 5529-5533. doi: $10.1073 /$ pnas.0810002106

Chiew, K. S., and Braver, T. S. (2013). Temporal dynamics of motivation-cognitive control interactions revealed by high-resolution pupillometry. Front. Psychol. 4:15. doi: 10.3389/fpsyg.2013.00015

Chiew, K. S., and Braver, T. S. (2014). Dissociable influences of reward motivation and positive emotion on cognitive control. Cogn. Affect. Behav. Neurosci. 14, 509-529. doi: 10.3758/s13415-014-0280-0

Chiew, K. S., and Braver, T. S. (2016). Reward favors the prepared: incentive and task-informative cues interact to enhance attentional control. J. Exp. Psychol. Hum. Percept. Perform. 42, 52-66. doi: 10.1037/xhp0000129

Cohen, M. X. (2017). Rigor and replication in time-frequency analyses of cognitive electrophysiology data. Int. J. Psychophysiol. 111, 80-87. doi: 10.1016/j.ijpsycho. 2016.02.001

Cohen, J. D., Braver, T. S., and Brown, J. W. (2002). Computational perspectives on dopamine function in prefrontal cortex. Curr. Opin. Neurobiol. 12, 223-229. doi: 10.1016/s0959-4388(02)00314-8

Cole, M. W., Reynolds, J. R., Power, J. D., Repovs, G., Anticevic, A., and Braver, T. S. (2013). Multi-task connectivity reveals flexible hubs for adaptive task control. Nat. Neurosci. 16, 1348-1355. doi: 10.1038/nn.3470

Costumero, V., Barrós-Loscertales, A., Bustamante, J., Fuentes, P., RosellNegre, P., Ventura-Campos, N., et al. (2015). A new window to understanding individual differences in reward sensitivity from attentional networks. Brain Struct. Funct. 220, 1807-1821. doi: 10.1007/s00429-014-0760-6

Dajani, D. R., and Uddin, L. Q. (2015). Demystifying cognitive flexibility: implications for clinical and developmental neuroscience. Trends Neurosci. 38, 571-578. doi: 10.1016/j.tins.2015.07.003

Dosenbach, N. U. F., Fair, D. A., Cohen, A. L., Schlaggar, B. L., and Petersen, S. E. (2008). A dual-networks architecture of top-down control. Trends Cogn. Sci. 12, 99-105. doi: 10.1016/j.tics.2008.01.001

Dosenbach, N. U. F., Fair, D. A., Miezin, F. M., Cohen, A. L., Wenger, K. K., Dosenbach, R. A., et al. (2007). Distinct brain networks for adaptive and stable task control in humans. Proc. Natl. Acad. Sci. U S A 104, 11073-11078. doi: $10.1073 /$ pnas.0704320104

Dosenbach, N. U. F., Visscher, K. M., Palmer, E. D., Miezin, F. M., Wenger, K. K., Kang, H. C., et al. (2006). A core system for the implementation of task sets. Neuron 50, 799-812. doi: 10.1016/j.neuron.2006.04.031

Etzel, J. A., Cole, M. W., Zacks, J. M., Kay, K. N., and Braver, T. S. (2015). Reward motivation enhances task coding in frontoparietal cortex. Cereb. Cortex 26, 1647-1659. doi: 10.1093/cercor/bhu327

Fröber, K., and Dreisbach, G. (2014). The differential influences of positive affect, random reward and performance-contingent reward on cognitive control. Cogn. Affect. Behav. Neurosci. 14, 530-547. doi: 10.3758/s13415-014-0259-x

Fröber, K., and Dreisbach, G. (2016). How performance (non-)contingent reward modulates cognitive control. Acta Psychol. 168, 65-77. doi: 10.1016/j.actpsy. 2016.04.008
Fröber, K., Raith, L., and Dreisbach, G. (2018). The dynamic balance between cognitive flexibility and stability: the influence of local changes in reward expectation and global task context on voluntary switch rate. Psychol. Res. 82, 65-77. doi: 10.1007/s00426-017-0922-2

García-García, I., Jurado, M. A., Garolera, M., Segura, B., Marques-Iturria, I., Pueyo, R., et al. (2013). Functional connectivity in obesity during reward processing. Neuroimage 66, 232-239. doi: 10.1016/j.neuroimage.2012.10.035

Gilbert, A. M., and Fiez, J. A. (2004). Integrating rewards and cognition in the frontal cortex. Cogn. Affect. Behav. Neurosci. 4, 540-552. doi: 10.3758/cabn. 4.4 .540

Gonthier, C., Macnamara, B. N., Chow, M., Conway, A. R., and Braver, T. S. (2016). Inducing proactive control shifts in the AX-CPT. Front. Psychol. 7:1822. doi: 10.3389/fpsyg.2016.01822

Goulden, N., Khusnulina, A., Davis, N. J., Bracewell, R. M., Bokde, A. L., Mcnulty, J. P., et al. (2014). The salience network is responsible for switching between the default mode network and the central executive network: replication from DCM. Neuroimage 99, 180-190. doi: 10.1016/j.neuroimage. 2014.05.052

Hall, M. G., Naughtin, C. K., Mattingley, J. B., and Dux, P. E. (2018). Distributed and opposing effects of incidental learning in the human brain. Neuroimage 173, 351-360. doi: 10.1016/j.neuroimage.2018.02.068

Hallquist, M. N., Geier, C. F., and Luna, B. (2018). Incentives facilitate developmental improvement in inhibitory control by modulating controlrelated networks. Neuroimage 172, 369-380. doi: 10.1016/j.neuroimage.2018. 01.045

Hefer, C., and Dreisbach, G. (2016). The motivational modulation of proactive control in a modified version of the AX-continuous performance task: evidence from cue-based and prime-based preparation. Motiv. Sci. 2, 116-134. doi: $10.1037 / \operatorname{mot} 0000034$

Hefer, C., and Dreisbach, G. (2017). How performance-contingent reward prospect modulates cognitive control: increased cue maintenance at the cost of decreased flexibility. J. Exp. Psychol. Learn. Mem. Cogn. 43, 1643-1658. doi: $10.1037 / x \operatorname{lm} 0000397$

Howe, M. W., Tierney, P. L., Sandberg, S. G., Phillips, P. E., and Graybiel, A. M. (2013). Prolonged dopamine signalling in striatum signals proximity and value of distant rewards. Nature 500, 575-579. doi: 10.1038/nature12475

Jaspar, M., Genon, S., Muto, V., Meyer, C., Manard, M., Dideberg, V., et al. (2014). Modulating effect of COMT genotype on the brain regions underlying proactive control process during inhibition. Cortex 50, 148-161. doi: 10.1016/j. cortex.2013.06.003

Jimura, K., Locke, H. S., and Braver, T. S. (2010). Prefrontal cortex mediation of cognitive enhancement in rewarding motivational contexts. Proc. Natl. Acad. Sci. U S A 107, 8871-8876. doi: 10.1073/pnas. 1002007107

Kamijo, K., and Masaki, H. (2016). Fitness and ERP indices of cognitive control mode during task preparation in preadolescent children. Front. Hum. Neurosci. 10:441. doi: 10.3389/fnhum.2016.00441

Kennerley, S. W., Behrens, T. E., and Wallis, J. D. (2011). Double dissociation of value computations in orbitofrontal and anterior cingulate neurons. Nat. Neurosci. 14, 1581-1589. doi: 10.1038/nn.2961

Kerns, J. G., Cohen, J. D., MacDonald, A. W., Cho, R. Y., Stenger, V. A., and Carter, C. S. (2004). Anterior cingulate conflict monitoring and adjustments in control. Science 303, 1023-1026. doi: 10.1126/science.1089910

Kim, D. I., Manoach, D. S., Mathalon, D. H., Turner, J. A., Mannell, M., Brown, G. G., et al. (2009). Dysregulation of working memory and default-mode networks in schizophrenia using independent component analysis, an fBIRN and MCIC study. Hum. Brain Mapp. 30, 3795-3811. doi: 10.1002/hbm.20807

Lamm, C., Pine, D., and Fox, N. (2013). Impact of negative affectively charged stimuli and response style on cognitive-control-related neural activation: an ERP study. Brain Cogn. 83, 234-243. doi: 10.1016/j.bandc.2013.07.012

Li, Y. O., Adali, T., and Calhoun, V. D. (2007). Estimating the number of independent components for functional magnetic resonance imaging data. Hum. Brain Mapp. 28, 1251-1266. doi: 10.1002/hbm.20359

Ličen, M., Hartmann, F., Repovš, G., and Slapničar, S. (2016). The impact of social pressure and monetary incentive on cognitive control. Front. Psychol. 7:93. doi: 10.3389/fpsyg.2016.00093

Liston, C., Watts, R., Tottenham, N., Davidson, M. C., Niogi, S., Ulug, A. M., et al. (2006). Frontostriatal microstructure modulates efficient recruitment of cognitive control. Cereb. Cortex 16, 553-560. doi: 10.1093/cercor/bhj003 
Locke, H. S., and Braver, T. S. (2008). Motivational influences on cognitive control: behavior, brain activation and individual differences. Cogn. Affect. Behav. Neurosci. 8, 99-112. doi: 10.3758/cabn.8.1.99

Maraver, M. J., Bajo, M. T., and Gomez-Ariza, C. J. (2016). Training on working memory and inhibitory control in young adults. Front. Hum. Neurosci. 10:588. doi: 10.3389/fnhum.2016.00588

Meda, S. A., Gill, A., Stevens, M. C., Lorenzoni, R. P., Glahn, D. C., Calhoun, V. D., et al. (2012). Differences in resting-state functional magnetic resonance imaging functional network connectivity between schizophrenia and psychotic bipolar probands and their unaffected first-degree relatives. Biol. Psychiatry 71, 881-889. doi: 10.1016/j.biopsych.2012.01.025

Meda, S. A., Stevens, M. C., Folley, B. S., Calhoun, V. D., and Pearlson, G. D. (2009). Evidence for anomalous network connectivity during working memory encoding in schizophrenia: an ICA based analysis. PLoS One 4:e7911. doi: 10.1371/journal.pone.0007911

Medford, N., and Critchley, H. D. (2010). Conjoint activity of anterior insular and anterior cingulate cortex: awareness and response. Brain Struct. Funct. 214, 535-549. doi: 10.1007/s00429-010-0265-x

Menon, V. (2011). Large-scale brain networks and psychopathology: a unifying triple network model. Trends Cogn. Sci. 15, 483-506. doi: 10.1016/j.tics.2011. 08.003

Menon, V., and Uddin, L. Q. (2010). Saliency, switching, attention and control: a network model of insula function. Brain Struct. Funct. 214, 655-667. doi: 10.1007/s00429-010-0262-0

Miller, E. K., and Cohen, J. D. (2001). An integrative theory of prefrontal cortex function. Annu. Rev. Neurosci. 24, 167-202. doi: 10.1146/annurev.neuro.24. 1.167

Nasr, S., Moeeny, A., and Esteky, H. (2008). Neural correlate of filtering of irrelevant information from visual working memory. PLoS One 3:e3282. doi: 10.1371/journal.pone.0003282

Nee, D. E., Jonides, J., and Berman, M. G. (2007). Neural mechanisms of proactive interference-resolution. Neuroimage 38, 740-751. doi: 10.1016/j.neuroimage. 2007.07.066

Nelson, S. M., Dosenbach, N. U. F., Cohen, A. L., Wheeler, M. E., Schlaggar, B. L., and Petersen, S. E. (2010). Role of the anterior insula in task-level control and focal attention. Brain Struct. Funct. 214, 669-680. doi: 10.1007/s00429-0100260-2

Niv, Y. (2007). Cost, benefit, tonic, phasic. Ann. N Y Acad. Sci. 1104, 357-376. doi: 10.1196/annals.1390.018

Papo, D., Buldú, J. M., Boccaletti, S., and Bullmore, E. T. (2014). Complex network theory and the brain. Philos. Trans. R. Soc. Lond. B Biol. Sci. 369:20130520. doi: 10.1098/rstb.2013.0520

Paxton, J. L., Barch, D. M., Racine, C. A., and Braver, T. S. (2008). Cognitive control, goal maintenance and prefrontal function in healthy aging. Cereb. Cortex 18, 1010-1028. doi: 10.1093/cercor/bhm135

Paxton, J. L., Barch, D. M., Storandt, M., and Braver, T. S. (2006). Effects of environmental support and strategy training on older adults' use of context. Psychol. Aging 21, 499-509. doi: 10.1037/0882-7974.21.3.499

Pochon, J., Levy, R., Fossati, P., Lehericy, S., Poline, J., Pillon, B., et al. (2002). The neural system that bridges reward and cognition in humans: an fMRI study. Proc. Natl. Acad. Sci. U S A 99, 5669-5674. doi: 10.1073/pnas.082111099

Polizzotto, N. R., Hill-Jarrett, T., Walker, C., and Cho, R. Y. (2018). Normal development of context processing using the AXCPT paradigm. PLoS One 13:e0197812. doi: 10.1371/journal.pone.0197812

Schultz, W. (1998). Predictive reward signal of dopamine neurons. J. Neurophysiol. 80, 1-27. doi: 10.1152/jn.1998.80.1.1

Seeley, W. W., Menon, V., Schatzberg, A. F., Keller, J., Glover, G. H., Kenna, H., et al. (2007). Dissociable intrinsic connectivity networks for salience processing and executive control. J. Neurosci. 27, 2349-2356. doi: 10.1523/JNEUROSCI. 5587-06.2007

Shenhav, A., Botvinick, M. M., and Cohen, J. D. (2013). The expected value of control: an integrative theory of anterior cingulate cortex function. Neuron 79, 217-240. doi: 10.1016/j.neuron.2013.07.007

Shenhav, A., Cohen, J. D., and Botvinick, M. M. (2016). Dorsal anterior cingulate cortex and the value of control. Nat. Neurosci. 19, 1286-1291. doi: 10.1038/ nn. 4384
Shirer, W. R., Ryali, S., Rykhlevskaia, E., Menon, V., and Greicius, M. D. (2012). Decoding subject-driven cognitive states with whole-brain connectivity patterns. Cereb. Cortex 22, 158-165. doi: 10.1093/cercor/bhr099

Small, D. M., Gitelman, D., Simmons, K., Bloise, S. M., Parrish, T., and Mesulam, M.-M. (2005). Monetary incentives enhance processing in brain regions mediating top-down control of attention. Cereb. Cortex 15, 1855-1865. doi: 10.1093/cercor/bhi063

Smith, S. M., Miller, K. L., Moeller, S., Xu, J., Auerbach, E. J., Woolrich, M. W., et al. (2012). Temporally-independent functional modes of spontaneous brain activity. Proc. Natl. Acad. Sci. U S A 109, 3131-3136. doi: 10.1073/pnas. 1121329109

Sridharan, D., Levitin, D. J., and Menon, V. (2008). A critical role for the right fronto-insular cortex in switching between central-executive and default-mode networks. Proc. Natl. Acad. Sci. U S A 105, 12569-12574. doi: 10.1073/pnas. 0800005105

Taylor, S. F., Welsh, R. C., Wager, T. D., Phan, K. L., Fitzgerald, K. D., and Gehring, W. J. (2004). A functional neuroimaging study of motivation and executive function. Neuroimage 21, 1045-1054. doi: 10.1016/j.neuroimage. 2003.10.032

Uddin, L. Q. (2014). Salience processing and insular cortical function and dysfunction. Nat. Rev. Neurosci. 16, 55-61. doi: 10.1038/nrn3857

Westbrook, A., and Braver, T. S. (2016). Dopamine does double duty in motivating cognitive effort. Neuron 89, 695-710. doi: 10.1016/j.neuron.2015. 12.029

Xu, J., Potenza, M. N., and Calhoun, V. D. (2013a). Spatial ICA reveals functional activity hidden from traditional fMRI GLM-based analyses. Front. Neurosci. 7:154. doi: 10.3389/fnins.2013.00154

Xu, J., Potenza, M. N., Calhoun, V. D., Zhang, R., Yip, S. W., Wall, J. T., et al. (2016). Large-scale functional network overlap is a general property of brain functional organization: reconciling inconsistent fMRI findings from general-linear-model-based analyses. Neurosci. Biobehav. Rev. 71, 83-100. doi: 10.1016/j.neubiorev.2016.08.035

Xu, J., Zhang, S., Calhoun, V. D., Monterosso, J., Li, C.-S. R., Worhunsky, P. D., et al. (2013b). Task-related concurrent but opposite modulations of overlapping functional networks as revealed by spatial ICA. Neuroimage 79, 62-71. doi: 10.1016/j.neuroimage.2013.04.038

Yee, D. M., and Braver, T. S. (2018). Interactions of motivation and cognitive control. Curr. Opin. Behav. Sci. 19, 83-90. doi: 10.1016/j.cobeha.2017. 11.009

Yip, S. W., Gross, J. J., Chawla, M., Ma, S. S., Shi, X. H., Liu, L., et al. (2018). Is neural processing of negative stimuli altered in addiction independent of drug effects? Findings from drug-naive youth with internet gaming disorder. Neuropsychopharmacology 43, 1364-1372. doi: 10.1038/npp. 2017.283

Zedelius, C. M., Veling, H., and Aarts, H. (2011). Boosting or choking-How conscious and unconscious reward processing modulate the active maintenance of goal-relevant information. Conscious. Cogn. 20, 355-362. doi: 10.1016/j.concog.2010.05.001

Zhang, H., Kang, C., Wu, Y., Ma, F., and Guo, T. (2015). Improving proactive control with training on language switching in bilinguals. Neuroreport 26, 354-359. doi: 10.1097/WNR.0000000000000353

Zhang, S., and Li, C. S. (2012). Functional networks for cognitive control in a stop signal task: independent component analysis. Hum. Brain Mapp. 33, 89-104. doi: $10.1002 / \mathrm{hbm} .21197$

Conflict of Interest Statement: The authors declare that the research was conducted in the absence of any commercial or financial relationships that could be construed as a potential conflict of interest.

Copyright (C) 2018 Qiao, Xu, Che, Zhang, Li, Xue, Li and Chen. This is an open-access article distributed under the terms of the Creative Commons Attribution License (CC BY). The use, distribution or reproduction in other forums is permitted, provided the original author(s) and the copyright owner(s) are credited and that the original publication in this journal is cited, in accordance with accepted academic practice. No use, distribution or reproduction is permitted which does not comply with these terms. 